
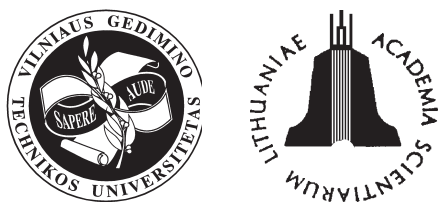

ISSN 1648-4142 print / ISSN 1648-3480 online TRANSPORT

www.transport.vtu.lt

\title{
SMOOTH TRAFFIC FLOW AS ONE OF THE MOST IMPORTANT FACTORS FOR SAFETY INCREASE IN ROAD TRANSPORT
}

\author{
Alica Kalašová ${ }^{1}$, Milan Stacho ${ }^{2}$ \\ Department of Road and Urban Transport, Faculty of Operation and Economics \\ of Transport and Communications, University of Žilina \\ E-mail:1 Alica.Kalasova@fpedas.utc.sk; ${ }^{2}$ Milan.Stacho@fpedas.utc.sk \\ Received 10 November 2005; accepted 4 January 2006
}

\begin{abstract}
The elimination of the connection between the increase of transport and economical growth has been the main aim of transport policy of the EU for a few years, which has not been accomplished yet. The volume of transport in the EU has been growing at more or less the same rate as economy or more, by nearly $20 \%$ with passenger transport and by approximately $30 \%$ with freight transport haulage. Transportation causes considerable external costs that although have got concrete originators, are not paid by them. The biggest external costs are connected with emissions, noise, accidents and congestions. In our contribution we would like to characterize congestions and possible solutions.
\end{abstract}

Keywords: external costs, traffic flows, intelligent transportation systems (ITS), ITS technologies, communication systems.

\section{Introduction}

One of the most significant factors affecting the increase of road transport safety is the reduction of external costs. External costs are described as follows.

When consumers decide to purchase an item or take a trip, they examine the price of a given option and compare it to the gain or satisfaction they expect to derive from the item or trip. For instance, an individual wishing to get from $A$ to $B$ will consider the price (use of public transport or his/her private car) and the quality of the service provided before choosing a given transport mode. Users are willing to accommodate a whole array of parameters (speed, frequent/regular service, quality, flexibility, etc.) in the transport price they pay.

Conversely, consumers of goods or services do not generally foot the full bill of the costs their decision imposes on society and environment. Such costs are defined as external because they are not reflected in the price paid by users and are not factors in the market. The main sources of external costs in the transport sector are accidents, congestion, air pollution, noise and climate change. Individuals using a given form of transport are not generally aware of the external costs generated and indeed it is possible that some of these costs have never been defined.

Nonetheless, external costs do exist and since they are not met by the parties responsible, they must be borne by society as a whole [1].

$\checkmark$ Significant external costs are: accidents, when transport systems are used, accidents occur generating a whole range of costs which are only partly covered by mutual risk insurance schemes (loss of life, medical care and disabilities sustained by victims, loss of production, etc.),

$\checkmark$ air pollution, emission of particulate matter, carbon monoxide, lead, volatile organic compounds, nitrogen oxides and sulphur dioxide, damaging health, environment and buildings,

$\checkmark$ climate change, greenhouse gases (mainly carbon dioxide $-\mathrm{CO}_{2}$ ) have an enduring impact on the earth's climate resulting in increased desertification, raised sea levels, serious harm to agriculture and other destructive environmental and health-related side-effect,

$\checkmark$ noise, transport generates noise which adversely affects humans in a variety of ways causing disturbances, stress and more serious health problems,

$\checkmark$ congestion, more vehicles are being added to already dense traffic flows, particularly car traffic flows, paralyzing the system and leading to substantial wastage for all users. Congestion makes the entire transport system inefficient.

In our contribution we would like to analyze the 
problem of congestions which currently bother drivers in the EU and their reduction is one of the priorities of the EU's transport policy.

\section{Impact of congestions on safety}

An individual highway crash is a rare, random, multifactor event preceded by a situation in which one or more persons failed to cope with their environment. In the aggregate, however, traffic crashes are quite numerous and often follow certain patterns that can be identified. Crashes reflect a shortcoming in one or more components of the driver-vehicleroadway system. It is therefore very important for freeway practitioners to monitor traffic collision experience and to use this information to identify, plan, implement, and evaluate corrective actions. Numerous approaches exist for improving safety and reducing crashes on highways. Many of these are beyond the scope of freeway management and operations, per se (e.g., enforcing seat belt laws, in-vehicle crashavoidance technologies, geometric realignment); but others - such as improved signing and lighting, skid resistance pavement, adding shoulders and auxiliary lanes, and removing obstacles - are well within the realm of "operations".

As previously noted, a major goal of freeway management and operations is to reduce congestion; and a reduction in congestion may also enhance safety. But how does congestion affect highway safety? The basic theory behind the interaction is that congestion leads to higher vehicle densities (i.e., more closely spaced vehicles on a roadway), which provides more opportunities for conflict. Congestion also reduces vehicle speeds, which implies that when vehicles are engaged in a crash, the collision forces are lower thus reducing the injury to occupants. Another aspect of the model is the concept of "secondary" crashes - crashes that occur due to conditions produced by an existing crash. Some of these conditions - which wouldn't exist without the occurrence of the first crash - include rapid backward queue formation (as vehicles suddenly stop to avoid the first crash), rubbernecking by drivers, and the manoeuvres of emergency vehicles. Finally, the flow restrictions produced by crashes worsen existing congestion.

The details of the relationship between congestion and safety are not well understood (with the exception of lower crash severities which have been documented in a general way for congested conditions and the associated lower speeds). Based on the limited work that has been performed, a few tentative conclusions may be drawn:

$\checkmark$ Crash potential probably increases as congestion increases.

$\checkmark$ There is a lower proportion of single vehicle crashes (e.g., run-off-road, rollover, collision with a fixed object) during congested conditions and a higher proportion of multiple vehicle crashes.

$\checkmark$ Crash severities (extent and nature of personal injuries) are lower during congested conditions due to lower vehicle speeds at the moment of crash impact.

In general, it can be assumed that any operational improvement that reduces congestion will lead to fewer crashes. The severity of crashes that occur will be higher, however, and it is likely that a greater proportion will be single vehicle crashes. Knowing these facts target mitigation strategies can single vehicle crashes and higher severities - such as wider roadside recovery zones, protection of highway "furniture," and coordination with emergency medical services. Moreover, operations philosophy must take a systems-oriented view where the consequences of a specific action (e.g., flow improvements) consider linked impacts such as safety.

\section{Traffic flow theory}

The generalized relationships between speed, density and flow rate are shown in Fig 1, with these parameters defined as follows [2].

$\checkmark$ Flow Rate - the equivalent hourly rate (vehicles per hour) at which vehicles pass over a given point or section of a lane or roadway during a given time interval of less than one hour.

$\checkmark$ Speed - defined as a rate of motion expressed as distance per unit of time, generally as kilometre per hour $(\mathrm{km} / \mathrm{h})$. In characterizing the speed of a traffic stream, a representative value must be used be-

Generalized relationships among speed, density and flows rate on uninterrupted - flows facilities
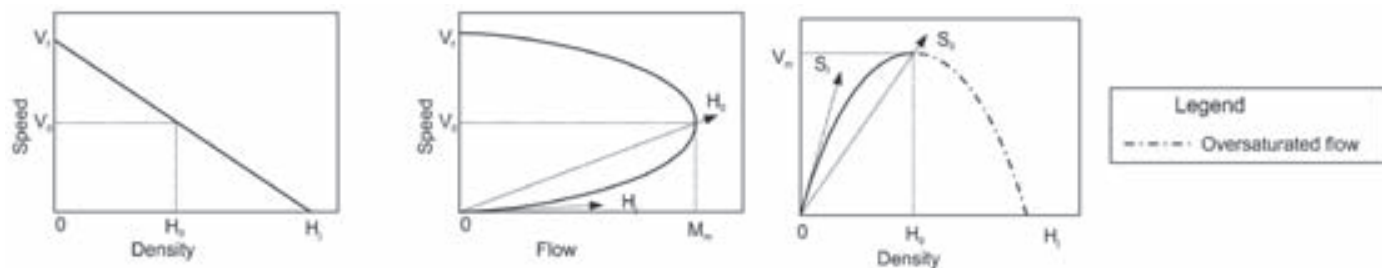

Fig 1. Basic traffic service 
cause broad distribution of individual speeds is observable in the traffic stream. The curves in Fig 1 utilize "average travel speed" which is computed by dividing the length of the highway segment under consideration by the average travel time of the vehicles traversing it.

$\checkmark$ Density - the number of vehicles occupying the given length of a lane or roadway at a particular instant. For the curves shown in Fig 1density is averaged over time and is usually expressed as vehicles per hour (veh/h).

The form of these curves depends on the prevailing traffic and roadway conditions on the segment under study. Moreover, while the diagrams in Fig 16 show continuous curves; in reality they are like discontinuities with a part of these curves not present. The curves illustrate the following significant points.

$\checkmark$ A zero flow rate occurs under two different conditions. One is when there are no vehicles on the facility - the density is zero, and the flow rate is zero. The second is when density becomes so high that all vehicles must stop - the speed is zero, and the flow rate is zero, because there is no movement and vehicles cannot pass a point on the roadway.

$\checkmark$ Between these two extreme points the dynamics of traffic flow produces a maximizing effect. As flow increases from zero, density also increases, since more vehicles are on the roadway. When this happens, speed declines because of the interaction of vehicles. This decline is negligible at low and medium densities and flow rates. As the density further increases, these generalized curves suggest that speed decreases significantly just before the capacity is achieved, with the capacity being defined as the product of density and speed resulting in the maximum flow rate. This condition is shown as optimum speed $V_{o}$ (often called critical speed), optimum density $M_{o}$ (sometimes referred to as critical density), and maximum flow $V_{m}$.

Efficient freeway operation depends on the balance between capacity and demand. In the simplest terms, highway congestion results when traffic demand approaches or exceeds the available capacity of the highway system. As vehicle demand approaches highway capacity, traffic flow begins to deteriorate. Flow is interrupted by spots of turbulence and shock waves which disrupt efficiency. Then traffic flow begins to break down rapidly followed by further deterioration of operational efficiency. The result of this spiralling inefficiency can be observed during every weekday commute in almost every metropolitan area. Drivers push their way onto already crowded freeways to join thousands of others already caught in seemingly endless traffic jams. Unfortunately, by joining the already impeded traffic flow, drivers become a part of the problem, creating even greater inefficiencies: more stop-and-go traffic conditions, longer delays and greater potential for accidents.

While this is a simple concept, traffic demand is not constant. It can vary significantly depending on the season of the year, the day of the week, and even the time of the day. Also, the capacity is not a constant - it can change (sometimes rapidly) because of weather, work zones, traffic incidents, or other events. It is not necessarily simple, either. The physical fact of finiteness and the principle of conservation underly traffic stream behaviour as reflected in the smooth curves in Fig 1. However, the actual performance of a particular section of a freeway at a particular point of time is more ambiguous, resulting from variations in individual human behaviour and the mix of vehicle types using the facility. It may be possible to predict the average behaviour and average capacity and the variances about these averages for a traffic stream, but never the precise behaviour.

The principles of macroscopic models are relations between the essential characteristics of a traffic flow - intensity, density (quantitative characteristics) and speed (a qualitative characteristic). For complete description only two of these characteristics are sufficient, the third one can be calculated according to the continuity equation:

$$
M=H \text {.V. }
$$

\section{Resolving of congestions using intelligent trans- portation systems}

One of the possible ways of increasing safety is to provide the participants of traffic with information in advance. Conditions for steady and safe traffic are made in this way. Intelligent transport systems - ITS are able to give us this possibility. Basic aim of ITS is to $[3,4]$ :

$\checkmark$ Increase the safety of road transport.

$\checkmark$ Increase the efficiency of transport represented by cut down transport time.

$\checkmark$ Increase the quality of environment.

$\checkmark$ Improve commercial productivity of a company.

ITS are systems which help to make efficient use of road and urban communication network using information, communication and directing technologies. They make basic conditions for high quality communication and information society that we are approaching also in our conditions.

Intelligent Transportation Systems (ITS) are the application of advanced electronics, computer, communications and sensor technologies - in an integrated manner - to increase the efficiency and safety 
of the surface transportation network. ITS encompass technologies that can lead to:

$\checkmark$ Better management and operations of the existing highway, public transportation and railroad infrastructure to ease congestion and respond to crises.

$\checkmark$ Safer and more convenient travel for people.

$\checkmark$ More efficient and secure freight movements.

Effectively used ITS open the door to new ways of managing, operating, expanding, refining, reconfiguring and use of transportation system. ITS have proven themselves as a significant enabler of freeway management and operations. Combined with ITS technologies:

Freeway Management System (FMS) consists of a set of resources (e.g., electronic systems, people, objects, and strategies) that are used to accomplish a set of goals to improve the operation of the freeway network. Other elements of an ITS-based freeway management system that support these functions include:

Surveillance and Detection Systems These devices collect information on traffic flow and roadway performance and allow operators to monitor conditions (in real time) on the freeway system. The sensors may collect data (volume, speed, travel time), or provide video images via cameras. The data collected feed the control and information dissemination functions and allow operators to intervene when appropriate into those functions. The data may also be stored (i.e., warehoused) for future analysis and performance evaluation. Sensor technology also allows the system to monitor roadway and environmental conditions such as pavement temperature and weather. Roadway and environmental condition information is often used in deciding how best to allocate resources for functions such as snow and ice control.

Transportation Management Centre. The computer systems and associated software, the user interfaces and operators themselves and associated resources are typically housed and operated from a traffic management centre although they can also be operated over a communication network without the need of a formal centre. In systems that include TMC, it serves as the information "nerve centre" for regional operations.

Communication Systems. The effective operation of all the functions mentioned above requires communication of data, video, and voice. Communication systems transmit data and video from the field to TMC or central location, transmit commands from TMC or central location to the field equipment, transmit information among agencies, distribute traveller information to the systems that disseminate it and allow personnel at any distance to communicate with one another.

From the point of view of ITS applications, positioning systems are among leading systems and deserve close attention. Considerable time and financial cut down should be pointed out. In this connection we should emphasize the necessity of creating uniform infrastructure of data gathering and distribution, creating a central system providing with current data from the given length of communications. This should be remembered while implementing master systems.

Global Navigation Satellite Systems. One of the most important technologies of ITS is satellite navigation which could influence the reduction of congestions.

At present there are two radio navigation satellite networks: the US GPS and the Russian Glonass systems, both designed during the Cold War for military purposes. Since the Russian system has not generated any civil applications, Galileo offers a real alternative to the de facto monopoly of GPS and the US industry. GPS is used to a large extent for civil purposes, but it does have several major shortcomings:

$\checkmark$ Mediocre and varying position accuracy (sometimes to only several dozen metres), depending on place and time.

$\checkmark$ Reliability leaves something to be desired. Regions at high latitudes crossed by many aviation routes do not have dependable coverage. Signal penetration in dense areas and town centres is unreliable. Furthermore, the predominantly military character of GPS means that there is always a risk of civil users being cut off in the event of a crisis.

Whether intentional or otherwise, signal interruptions can have disastrous consequences, especially as there is no warning and no immediate information about errors [3].

There have been signal cut-offs of a few minutes during GPS operation, which lead the system provider to the adaptation to civil needs and the necessity of standards development for the utilization of satellite navigation in civil aviation. If these standards meet aviation demands they will suit also other users. Standard and recommended procedures - SARP, which are focused on accuracy, integrity and time to alery, continuity and availability, have been passed.

These standards lead to the building of augmentation systems such as American WAAS (wide area augmentation system), Japanese MSAS (MTSAT satellite augmentation system), European services EGNOS (European geostationary navigation overlay service) and even probably Chinese satellite system Beidou [3]. 
Inspire of these standards they are only services based on originally military GPS of Glonass satellite systems.

The European Union (EU) therefore decided, in close cooperation with the European Space Agency (ESA), to develop a system of its own that meets the criteria for accuracy, reliability and security.

Galileo comprises a constellation of 30 satellites divided between three circular orbits at an altitude of around $24000 \mathrm{~km}$ to cover the earth's entity surface. They will be supported by a worldwide network of ground stations.

Galileo offers superior and constant accuracy thanks in particular to the structure of its satellite constellation and a ground relay system. Guaranteed accuracy to $1 \mathrm{~m}$ is necessary for certain applications such as entering a seaport or guiding a vehicle into parking space.

\section{Conclusion} that:

In the conclusion we would like to emphasize

$\checkmark$ Any definition needs to be understandable to the general public or to the elected officials to be useful. Congestion occurs and is caused by bottlenecks. All other mechanisms for describing traffic flow are related to measures of effectiveness.

$\checkmark$ The speed-flow relationship is fundamental to traffic theory.

$\checkmark$ A metrics for congestion is total trip time at posted speeds compared to total trip time at operating speed and an accompanying index for setting service standards."

$\checkmark$ Transportation congestion has local, cultural and time-of-duration components that defy strict terminology.

$\checkmark$ Congestion will always be relative.

With respect to the last point, congestion is typically viewed by travellers relative to their normal dayto-day experiences. Travellers accustomed to low speeds and congestion delays for 12 hours each day may not consider 10 minutes of delay per trip a problem. These travellers have learned to budget extra time or find other ways to cope with the delay.

A major goal of freeway management and operations is to keep freeway capacity and the vehicular demand on a freeway in balance. The most effective way to combat congestion is to take action before traffic flow deteriorates and congestion forms. It would be ideal to manage the demand on the freeway to prevent traffic flow from ever breaking down and congestion from forming. This is usually not possible and the best result is to delay the onset of con- gestion and speed the recovery from congestion, therefore minimizing the inefficiencies that congestion causes.

\section{References}

1. Schreyer, Ch.; Schneider, Ch. External costs of transport update study, International Railway Union, October 2004, Paris. ISBN 2-7461-0891-7.

2. Kalašová, A.; Stacho, M. Decrease in congestions is one of the most important factors for increase in safety in road transport. In: Modern Safety Technologies in Transportation, 2005, Košice. ISBN 80-969106-1-2.

3. Kevický, D. - Kalašová, A. Satelite navigation system, EDIS - vydavatel'stvo ŽU, 2004. ISBN 80-8070-295-0 (in Slovak).

4. European Commision, ESA: GALILEO Mission High Level Definition, September 2002. 